\title{
Evaluation of the Success Rate of Emergency Cerclage by Double- Suture Method in Pregnant Women With the Prolapsed Amniotic Membrane
}

\author{
Ali Emami \\ Qazvin University of Medical Sciences \\ Masumeh Dadashaliha ( $\square$ m.dadashaliha@qums.ac.ir) \\ Qazvin University of Medical Sciences
}

\section{Research Article}

Keywords: cerclage, cervical incompetence, fetal death, premature birth

Posted Date: November 15th, 2021

DOI: https://doi.org/10.21203/rs.3.rs-1012739/v1

License: (c) (i) This work is licensed under a Creative Commons Attribution 4.0 International License.

Read Full License 


\section{Abstract}

\section{Background}

Cervical insufficiency is a risk factor for preterm labor and second trimester loss. Cervical cerclage is the choice treatment for women with this risk factor. An emergency cerclage is recommended in cases with the prolapsed amniotic membrane at the dilated cervical os before 24 weeks of gestation. The aim of this study is to report our method with double-suture in emergency cerclage.

\section{Methods}

This is a cross-sectional study conducted between March 2017 and February 2019 of women who received the double-suture cerclage in Qazvin referral Kosar hospital. The inclusion criteria were women with gestational age of 18-24 weeks with the prolapsed amniotic membrane and cervical dilatation (more than $3-4 \mathrm{~cm}$ ) in the speculum exam. The exclusion criteria were the mother's dissatisfaction to do this cerclage, previous failure cerclage, twin pregnancy, preterm rupture of membrane (PROM) signs and chorioamnionitis signs.

Results

Five women were included. All of them presented prolapsed membranes at or beyond the cervical os. The double-suture cerclage was performed for all of the patients. All of women delivered by normal vaginal delivery and their infants had no problems.

\section{Conclusions}

Despite the small number of the patients, this study showed that this method of cerclage was effective in pregnancy for women with high risk of preterm labor and second trimester loss. This technique requires an expert and trained surgical team. The following studies should be performed with a big population and compare with other methods of emergency cerclage.

\section{Introduction}

One of the major causes of perinatal mortality and morbidity are spontaneous preterm labors. Preterm births negatively affects on mother and child development. The preterm delivery rates are increasing in all over the world even in high-income countries and this rate was estimated $9.5 \%$ in the United States and $7.5 \%$ in France $(1,2)$.

The prominent etiologic factor in the second trimester preterm labor is cervical insufficiency which occurs in $0.5-1 \%$ of all pregnancies. The most common reason of cervical insufficiency is the incompetence of the cervix to maintain a pregnancy until 38 weeks (3). 
The American College of Obstetricians and Gynecologists defines cervical insufficiency as the inability of the cervix to retain a pregnancy in the second trimester in the absence of contractions, labor or both (4). Cervical cerclage is the only treatment for women with a history of cervical incompetency. The types of cerclage consist of prophylactic, therapeutic and emergency. The prophylactic type is done at the end of the first trimester for women who have history of three preterm birth or fetal loss. The therapeutic form is done for women who had cervical length $<25 \mathrm{~mm}$ during transvaginal ultrasonographing follow-up. The emergency type is done during the second trimester for the patient who have prolapsed membranes with dilated cervical os before 24 weeks of gestational age (2).

The efficacy of emergency cerclage is evaluated in one randomized clinical trial. Althuisius et al. study revealed that emergency cerclage can reduce the risk of preterm birth and neonatal morbidity compared with bed rest alone (5). Since the 1950s, cerclage techniques have been examined. The first one is the Shirodkar type which includes in the dissection of the vesico-cervical junction and using a suture close to the internal cervical os (6).

Then, McDonald presented a new technique with a simple string suture was inserted at cervicovaginal junction. The good point of this technique was no bladder dissection (7). These techniques are the most common routine techniques.

Bensen and Durfee presented a cervico-isthmic cerclage by the laparotomy (8). Laparoscopic and vaginal techniques were developed to decrease the morbidity of old procedures (9). Fernandez et al. described a cervico-isthmic cerclage associated with a polypropylene sling by the vaginal pathway. This technique is done in the first trimester and revealed a median gestational age at birth of 37.2 weeks and neonatal survive rate of $94 \%$. The Gynecologists use this cerclage in case of a history of failure of other cerclage techniques in previous pregnancies or in cases with absent portio vaginalis of cervix (10).

Herein, we aim to report a new successful cerclage technique which was done in 5 cases. The uniqueness of our cases are performing that this cerclage with the help of a Foley catheter and a double suture method in pregnant women with the prolapsed amniotic membrane and need to emergency cerclage.

\section{Materials And Methods}

This cross-sectional study was performed between March 2017 and February 2019 in Qazvin referral Kosar hospital.

The inclusion criteria were women with gestational age of 18-24 weeks which referred to hospital with the prolapsed amniotic membrane and cervical dilatation (more than $3-4 \mathrm{~cm}$ ) in the speculum exam. The exclusion criteria were the mother's dissatisfaction to do this cerclage, previous failure cerclage, twin pregnancy, preterm rupture of membrane (PROM) signs and chorioamnionitis signs.

At admission, a researcher performed gynecological exam using the speculum to see cervical os and evaluate amniotic membrane. An ultrasound was done to check fetal vitality and amniotic fluid index 
(AFI). Paraclinical tests (differential complete blood count, C - reactive protein and erythrocyte sedimentation rate) and checking clinical signs (tachycardia, tachypnea, fever, uterine contraction and positive vaginal discharge) was done for roll out chorioamnionitis.

Demographic data were collected with a checklist includes age, height, weight, body mass index (BMI), gravidity, parity, gestational age, abortion history, preterm labor history, cerclage history, past medical history, past surgical history and drug history.

After obtaining the patient's informed consent about the possibility of rupturing of the amniotic membrane and the risk of fetal death, we begin to perform a new method of cerclage.

\section{Surgical technique}

The procedure is performed under general anesthesia. A prophylactic antibiotic treatment by ceftriaxone $1 \mathrm{gr}$ and azithromycin $1 \mathrm{gr}$ was used before the procedure. The suppository of indomethacin $100 \mathrm{mg} / \mathrm{BD}$ as tocolytic for 48 hours after the process was described. The suppository of progesterone (Cyclogest) $400 \mathrm{mg}$ was ordered before surgery. Surgery is performed after exclusion the clinical and paraclinical signs of a septic consent. The patient is placed in the Trendlenberg position in the operating room. The gynecologist passes Foley catheter No. 18 through to the opening of the cervix and by filling the Foley balloon with $50 \mathrm{cc}$ of normal saline, we gently guide the amniotic membrane upwards to the uterine cavity. This procedure prevents the needle collision to amniotic membrane during cerclage. Then the gynecologist does cerclage associated with Mersilene polyester fiber suture in two steps, around the internal os and around the external os. Before ending suturing, the Foley balloon is gently drained and the catheter is removed. Then, the sutures of the internal and external os were tied. After that gynecologist tights the cerclage knot and removes the patient from the Trendlenberg position (Figure 1).

After surgery, the patients receive a non-steroidal anti-inflammatory drug (the suppository of indomethacin $100 \mathrm{mg} / \mathrm{BD}$ for 48 hours) and have to rest. The antibiotic treatment by azithromycin 500 $\mathrm{mg} /$ daily and metronidazole $500 \mathrm{mg} / \mathrm{BD}$ was prescribed for one week after surgery. The ampule of hydroxyprogesterone caproate (proluton) $250 \mathrm{mg} / \mathrm{IM}$ weekly was prescribed. After discharge from hospital, the gynecologist taught the patient alarm signs for infection (vaginal bleeding, rupture of membrane, tachycardia, tachypnea, fever, abdominal pain and odor discharge) to refer to hospital if they sign occur. The doctor visited the patient weekly and checked fever, the paraclinincal tests (CBC, diff, ESR and CPR) for infection and ultrasound for evaluation fetal vitality. If alarm signs occur or uterine contractions begin, the gynecologist will terminate the pregnancy. In follow up, the betamethasone $12 \mathrm{mg}$ for 2 days was described after 24 weeks of gestational age for all cases to induce lung maturity

\section{Results}

During the study period, 5 emergency cerclage for prolapsing amniotic membrane were performed. We will describe them: 


\section{Case1}

The first case was a 28-year-old woman with no living child. She had a history of abortion at 8 weeks of gestation with dilation and curettage. She had no medical history. She had a history of secondary infertility 10 years. She was referred to hospital with hypogasteric pain. Her vital sign was stable. In the speculum exam, she had prolapsed amniotic membranous and cervical dilation 3-4 cm. At 18 weeks gestational age were diagnosed dilated cervix with prolapsed membranous beyond the cervical os. She had no sign of infection. Her laboratory tests were normal. The new emergency cerclage technique was performed for the patient and it was successful. The women delivered at 39 weeks by normal vaginal delivery. Her infant was normal with Apgar 9.

\section{Case 2}

The second case was a 31-year-old woman with no living child. She had a history of abortion at 6 weeks of gestation and ectopic pregnancy in past pregnancies. She had a history of 4 years infertility. She had a history of laparoscopy and salpingostomy for an ectopic pregnancy. A day before of referring, in her screening and anomaly scan sonography, the radiologist reported funneling cervix with cervical length 22 $\mathrm{mm}$. At 18 weeks and 5 days of gestational age, she was referred to hospital for prophylactic cerclage. Her vital sign was stable. Before the procedure and in the speculum exam, she had cervical dilation (3-4 $\mathrm{cm}$ ) and was diagnosed with prolapsed membranous beyond the cervical os. The prophylactic cerclage was changed to emergency cerclage. She had no sign of infection. Her laboratory tests were normal. The new emergency cerclage technique was performed for the patient and it was successful. The women delivered at 36 weeks by normal vaginal delivery.

\section{Case 3}

The third case was a 19-year-old woman with no living child. She had a history of miscarriage at 16 weeks of gestation with dilation and curettage. She had no history of infertility. In her screening and anomaly scan sonography, the radiologist reported with cervical length $19 \mathrm{~mm}$. In the same day and at 18 weeks and 5 days of gestational age, she was referred to hospital for prophylactic cerclage. Her vital sign was stable. Before the procedure and in the speculum exam, she had cervical dilation $3 \mathrm{~cm}$ and was diagnosed with prolapsed membranous beyond the cervical os. She had no sign of infection. Her laboratory tests were normal. The prophylactic cerclage was changed to emergency cerclage. The new emergency cerclage technique was performed for the patient and it was successful. The women delivered at 37 weeks and 5 days by normal vaginal delivery.

\section{Case 4}

The 4th case was a 37-year-old woman with no living child. She had a history of 2 abortions and preterm labor. She had a history of infertility for 10 years. She was referred with the prolapsed amniotic sac at gestational age of 21 weeks. Her vital sign was normal. In the speculum exam, the amniotic sac was seen in the vagina. In her anomaly scan sonography, the radiologist reported that the cervical length is $30 \mathrm{~mm}$. 
She was under treatment of proluton weekly. She had no sign of infection. Her laboratory tests were normal. The new emergency cerclage technique was performed for the patient and it was successful. The women delivered at 31 weeks and 6 days by normal vaginal delivery. The neonate was in neonatal intensive care (NICU) for 10 days and then discharged healthy.

\section{Case 5}

She was a 37-year-old woman with one living boy. She had a history of one abortion lower at 6 weeks of gestational age. She had no history of preterm labor. She had a history of secondary infertility 6 years. In her screening sonography, the radiologist reported cervical length $30 \mathrm{~mm}$. She was referred to hospital with dull abdominal pain at 19 weeks and 4 days of gestational age. Her vital sign was stable. In the speculum exam, she had cervical dilation. She was diagnosed with prolapsed membranous beyond the cervical os. She had no sign of infection. Her laboratory tests were normal. The new emergency cerclage technique was performed for the patient and it was successful. The women delivered at 36 weeks and 3 days by normal vaginal delivery.

All women received antibiotic before and after surgery. None of them had any signs of infections and surgical complications.

\section{Discussion}

This paper reports on 5 cases of successful emergency cerclage by double-suture method among women who presented with the prolapsed amniotic membrane. This type of cerclage was effective in pregnancy prolongation for those four women in spite of the poor prognostic situation.

Emergency vaginal cervico-isthmic cerclage was a new method which was reported in Szmulewicz et al. study. In this method, prolapsed membranes gently pushed back into the uterine and then anterior semicircular colpotomy at the cervico-vaginal junction was done. A non-absorbable sling around cervicoisthmic junction was inserted and was tightened.

The modified McDonal cerclage was presented in Saremi et al. study. In this method, the suture of modified was inserted in the highest region of cervix. As much as possible, the bladder and vagina the bladder are elevated which can be kept out of damage to sutures. A semicircle was formed around the anatomical border of the bladder and was sutured without entering the bladder. Cerclage is performed at its highest level and near the kettle to the inner os (11). In our method, we used double sutures. The first suture was same as modified McDonald cerclage; the upper or right anterolateral and then the left anterolateral (2 and 10 o'clock) were carefully sutured. In the next step, external os was sutured. For decreasing the rate of rupture of the amniotic membrane and the bladder, the filling Foley catheter through cervix led the amniotic membrane into the uterine cavity.

The prophylactic cerclage was performed in Capmas et al. study. They performed vaginal cervico-isthmic cerclage for the patient $s$ with previous failed McDonald cerclage. Cervico-isthmic cerclage is inserted 
higher than other types of cerclages above the internal cervical orifice. They reported no significantly different between classic cerclage and vaginal cervico-isthmic cerclage group and their costs. The other point was the higher risk of fetal death and preterm labor delivery in vaginal cervico-isthmic cerclage method (12).

Szmulewicz et al. highlighted the emergency vaginal cervico-isthmic cerclage method. They reported 3 cases of emergency vaginal cervico-isthmic cerclage among the patient s who presented cervical modifications which showed with ultrasound. The study revealed emergency vaginal cervico-isthmic cerclage can be performed without any complication or fetal loss (2). In this study, the amniotic membranes of all cases was prolapsed to the vagina, the external os was open, the cervix was dilated, the amniotic membrane was seen in the vagina and emergency double-suture cerclage associated with using of filled Foley catheter. All of the fetus was born without any complications and they continue to live.

Another type of emergency cerclage which performed in Tanaka et al. study, was bridge suture for the successful McDonald cerclage. The first suture was inserted in internal os as bridge suture then McDonald cerclage was performed. They reported the first bridge suture without any complications, fatal loss and preterm labor (13).

For comparison between emergency cerclage and bed rest in the patient presented with amniotic sac prolapsed, Stupin et al. study was performed. They used the combination of double cerclage (Branch/Hochuli) and complete closure of the uterus opening (Szendi/Saling). Finally, they reported the operative management had longer pregnancy, higher birth weight and lower prenatal mortality (14).

This method of cerclage showed that double-suture associated with Foley catheter can be performed with hopeful results. This technique requires a trained surgeon. For evaluating the results and complications of this method and comprising with other methods, more clinical studies should be performed.

\section{Declarations}

\section{Acknowledgments}

The authors would like to thank the Clinical Research Development Unit of Kosar Hospital and the student research committee of Qazvin University of Medical Sciences.

\section{Authors' contributions}

Concept; MD, Design; MD, Data Collection or Processing; MD \& AE, Analysis; AE, Literature Search; AE, Writing; $A E$

\section{Funding}

None.

\section{Availability of data and materials}


The datasets used during the current study are available from the corresponding author on reasonable request

\section{Ethics approval and consent to participate}

Before the patient s were enrolled in this study, informed consents were obtained by participant. This study was approved by the Research Ethics Committee of Qazvin Faculty of Medical Sciences (Code: IR.QUMS.REC.1399.247). All experiments were performed in accordance with relevant guidelines and regulations.

\section{Consent for publication}

Not applicable.

\section{Competing interests}

The authors declare that they have no competing interests.

\section{Author details}

${ }^{1}$ Student Research Committee, Qazvin University of Medical Sciences, Qazvin, Iran. ${ }^{2}$ Assistant professor, Clinical Research Development Unit, Kosar Hospital, Qazvin University of Medical Sciences, Qazvin, Iran

\section{References}

1. Beauquier-Maccotta B, Mériot ME, Missonnier S, Shulz J, Soubieux M-J, De Wailly D. Le deuil périnatal: Clinique, pratiques et dispositifs thérapeutiques: Elsevier Health Sciences; 2020.

2. Szmulewicz C, Neveu M-E, Vigoureux S, Fernandez H, Capmas P. Emergency vaginal cervicoisthmic cerclage. Journal of gynecology obstetrics and human reproduction. 2019;48(6):391-4.

3. Prévention de l'accouchement prématuré par cerclage du col de l'utérus. Journal de Gynécologie Obstétrique et Biologie de la Reproduction. 2016;45(10):1299 - 323.

4. Obstetricians ACo, Gynecologists. ACOG Practice Bulletin No. 142: Cerclage for the management of cervical insufficiency. Obstetrics and gynecology. 2014;123(2 Pt 1):372.

5. Cervical incompetence prevention randomized cerclage trial: Emergency cerclage with bed rest versus bed rest alone. American Journal of Obstetrics and Gynecology. 2003;189(4):907 - 10.

6. Shirodkar V. A new method of operative treatment for habitual abortions in the second trimester of pregnancy. Antiseptic. 1955;52:299-300.

7. McDONALD IA. Suture of the cervix for inevitable miscarriage. BJOG: An International Journal of Obstetrics \& Gynaecology. 1957;64(3):346-50. 
8. BENSON RC, DURFEE RB. Transabdominal cervicouterine cerclage during pregnancy for the treatment of cervical incompetency. Obstetrics \& Gynecology. 1965;25(2):145-55.

9. Lesser KB, Childers JM, Surwit EA. Transabdominal cerclage: a laparoscopic approach. Obstetrics \& Gynecology. 1998;91(5):855-6.

10. Deffieux X, De Tayrac R, Louafi N, Gervaise A, Sénat M, Chauveaud-Lambling A, et al. Transvaginal cervico-isthmic cerclage using polypropylene tape: surgical procedure and pregnancy outcome: Fernandez's procedure. Journal de gynecologie, obstetrique et biologie de la reproduction. 2006;35(5 Pt 1):465-71.

11. Saremi A, Mirfenderesky A, Pooladi A. Comparison of Clinical Outcomes of the Treatment Cerclage between the Classical McDonald and the Sarem Modified McDonald Techniques. Sarem Journal of Reproductive Medicine. 2019;3(1):13-7.

12. Capmas P, Letendre I, Leray C, Deffieux X, Duminil L, Subtil D, et al. Vaginal cervico-isthmic cerclage versus McDonald cerclage in women with a previous failure of prophylactic cerclage: $A$ retrospective study. European Journal of Obstetrics \& Gynecology and Reproductive Biology. 2017;216:27-32.

13. Tanaka M, Hori Y, Shirafuji A, Kato M, Kato J, Kobayashi H, et al. Bridge Suture for Successful McDonald Emergency Cerclage. Gynecologic and obstetric investigation. 2017;82(5):446-52.

14. Stupin JH, David M, Siedentopf J-P, Dudenhausen JW. Emergency cerclage versus bed rest for amniotic sac prolapse before 27 gestational weeks: a retrospective, comparative study of 161 women. European Journal of Obstetrics \& Gynecology and Reproductive Biology. 2008;139(1):32-7. 